\title{
VALIDEZ, FIABILIDAD Y ESPECIFICIDAD DE LAS PRUEBAS DE AGILIDAD
}

\section{VALIDITY, RELIABILITY AND SPECIFITY OF AGILITY TESTS}

Yennys González De los Reyes ${ }^{1}$

\section{RESUMEN}

Los deportistas de actividades intermitentes requieren, dentro de la competencia, perseguir o eludir contrarios, dar respuesta a movimientos con cambios de velocidad y dirección provenientes de una variedad de estímulos; todo esto, de manera rápida, precisa y eficaz. El propósito de este estudio fue analizar la validez, la fiabilidad y la especificidad de las pruebas de agilidad y su relación con la velocidad. Adicionalmente, analizar factores de lateralidad e índices de masa corporal. Participaron 41 estudiantes de la Universidad de Ciencias Aplicadas y Ambientales U.D.C.A, 17 futbolistas, 10 voleibolistas y 14 estudiantes de la facultad de Ciencias del Deporte. Todos fueron valorados antropométricamente y realizaron cuatro pruebas: tres de agilidad, carrera de obstáculos (general), T-test (voleibol), Buttifant (fútbol) y, una de velocidad (30m). Las pruebas de agilidad, se realizaron por derecha e izquierda. Cada grupo repitió la prueba específica. Entre los resultados destacamos que la única prueba de agilidad válida fue la de Buttifant, ya que presentó independencia de la velocidad $(r=0,42)$ indicando que la validez de las pruebas depende del concepto de agilidad y de las características de cada deporte. La fiabilidad fue muy alta para obstáculos y Buttifant $(r>0,90)$, pero no aceptable para T-test $(\mathrm{r}=0,79)$. Se demostró que la prueba de Buttifant es más específica para los futbolistas, el T-test para los voleibolistas y, el de obstáculos, inespecífico. Además, el índice de masa corporal, no discriminó entre los grupos (n.s $=\mathrm{p}<0,05)$ y no se estableció influencia de lateralidad al no encontrarse diferencias significativas.

${ }^{1}$ Licenciada en Educación Física, Ms. en Voleibol. C. Dr. Ciencias de la Actividad Física y el Deporte. Docente investigadora, facultad Ciencias del Deporte. Universidad de Ciencias Aplicadas y Ambientales U.D.C.A. yengonzalez@udca.edu.co.
Palabras clave: Agilidad, prueba, deporte, cambios de dirección, entrenamiento.

\section{SUMMARY}

Sportsmen of intermittent activities require, within the competition, to persecute or to elude opponents and to respond to movements with changes of speed and direction of a variety of stimuli, in a fast, precise and effective form. The intention of this study was to analyze the validity, reliability and specificity of agility tests and its relation to speed. In addition, factors of laterality and the corporal mass index were analyzed. Participated 41 students of the Universidad de Ciencias Aplicadas y Ambientales U.D.C.A, 17 soccer players, 10 voleibol players and 14 students of the faculty of Sport Sciences. All were anthropometrically valued and three tests of agility were applied: race of obstacles (general), T-test (volleyball), Buttifant (soccer) and 30m speed. The agility tests were made from right and left. Each group made a post-test of the specific one. Among the results we emphasize that the only valid agility test was the Buttifant, since it presented speed independence $(r=0.42)$, showing that the tests validity depends on the concept of agility and the characteristics of each sport. The reliability of the test was very high for obstacles and Buttifant $(r>0.90)$, but not acceptable for T-test $(r=0.79)$. The present study demonstrated that the Buttifant test is more specific for soccer players, the T-test for volleyball players, and the obstacles test nonspecific. In addition, the corporal mass index did discriminate between the groups $(n . s=p<0.05)$ and laterality influence was not established due to the absence of significative differences.

Key words: Agility, test, sport, direction change speed, training. 


\section{INTRODUCCIÓN}

La agilidad se ha definido, clásicamente, como la habilidad de cambiar la dirección rápidamente (Bloomfield et al. 1994), pero también la habilidad de cambiar la dirección rápidamente y con precisión (Barrow E McGee, 1971). También es considerada como resultante de las capacidades físicas y de las perceptivo motrices (Porta, 1988; Hernández Corvo, 1989; Castañer, 1991; Sánchez Bañuelos (2002) citados por Fernández et al. 2006), además de un rápido movimiento de todo el cuerpo con cambio de dirección de velocidad en respuesta a un estímulo (Sheppard $\mathcal{E}$ Young, 2006). Dentro de las investigaciones que inicialmente relacionaron la velocidad lineal con la agilidad, se destaca la de Draper E Lancaster (1985), quienes reportaron una correlación, significativamente, baja a moderada $(r=0,472)$. Young et al. (1996) también evaluaron las relaciones entre la velocidad y los cambios de dirección de la velocidad (COD), en los jugadores de fútbol australianos, evidenciando débiles relaciones entre la habilidad para correr en línea recta y aquella para desempeñar tareas complejas, como driblar un balón (Tsitskarsis et al. 2003) o rebotar en el fútbol australiano (Young et al. 1996). Basándose en estas consideraciones, las pruebas y el entrenamiento de la agilidad, se dirigen hacia la demanda de habilidades que podrían aumentar la validez. Usando pruebas similares con cambios planeados, Baker (1999) examinó las diferencias de desempeño de jugadores élite de la liga de rugby. Los resultados del estudio de Baker (1999) apoyan aquellos de Young et al. (1996), en que los sprints y la agilidad son cualidades físicas separadas.

Djevalikian (1993) citado por Sheppard \& Young (2006) analizó la relación entre los desequilibrios de fuerza en la potencia muscular concéntrica entre la pierna derecha e izquierda y los COD, mientras se aparta la pierna más débil; en otras palabras, si un individuo es más débil de la pierna izquierda, se movería más despacio hacia la derecha, debido a la acción de empuje de la pierna izquierda. Los resultados sugirieron que no hubo relación significativa entre la potencia del músculo concéntrico y el COD. Sin embargo, Young et al. (2002) informaron que los desequilibrios musculares de las extremidades inferiores influyen en el COD. Se encontró que sus participantes eran, significativamente, más lentos para cambiar de dirección con la pierna más débil cuando se realiza una prueba unilateral de salto de caída para la fuerza reactiva.
Teóricamente, factores como la grasa del cuerpo y las longitudes de segmentos corporales pueden contribuir al desempeño de la agilidad. Las baterías de pruebas han revelado que atletas en deportes, tales como el rugby y el fútbol, quienes se desempeñan mejor en las pruebas COD, también tienden a tener más baja grasa en el cuerpo (Gabbett, 2002; Meir et al. 2001; Reilly et al. 2000). Otro estudio que involucró las correlaciones de grasa corporal y COD en jugadores de rugby encontró que las dos variables no estaban fuertemente correlacionadas $(r=0,21)$ (Webb \& Lander, 1983). La relación entre estas dos variables no es clara. Según Bouchard (2000), en su revisión sobre composición corporal una de las limitaciones del índice de masa corporal (IMC), como las tablas de talla y peso, fallan al considerar la proporción de la composición corporal, como el más importante componente en la distribución de la grasa corporal.

La prueba de agilidad Illinois, considerada una prueba estándar de agilidad, el sprint de $20 \mathrm{~m}$, la prueba upand-back y la prueba 505 fueron comparados. Los investigadores concluyeron que la prueba 505 fue la más válida de las pruebas examinadas, porque produjo la más alta correlación con la aceleración en la fase de cambio de la prueba, pero no se correlacionó altamente con la velocidad (Hastad E Lacy, 1994, citado por Gabbett, 2002).

El tema común en las pruebas de agilidad usadas por Baker (1999), Draper \& Lancaster (1985), Webb $\mathcal{E}$ Lander (1983) y Young et al. $(1996,2002)$ es que no existe ningún estímulo presente $\mathrm{y}$, por consiguiente ninguna de estas pruebas requiere algún componente cognoscitivo y reactivo. Dentro de la revisión de la literatura, se encontró un gran interés en la batería de pruebas encaminada al descubrimiento y predicción de talentos deportivos. Esto nos puede sugerir acerca de la importancia de la especificidad de las pruebas en la detección de talentos.

En el deporte de voleibol, Gabbett et al. (2006) analizaron los efectos de un programa de entrenamiento enfocado a las habilidades, la agilidad (T-test) y las habilidades técnicas en jugadores talentos de voleibol antes y después del programa propuesto. Se observaron mejoras importantes en la velocidad y la agilidad; esto puede reflejar la naturaleza altamente repetitiva de las habilidades técnicas del voleibol (bloqueo y remate). Continuando con este deporte, Barnes et al. (2007) 
concluyeron que los individuos con gran salto en contra movimiento, también poseen tiempos rápidos en las pruebas de agilidad y sugiere un entrenamiento especifico en salto vertical para incrementar diferentes tipos de agilidad.

Uno de los deportes que más se ha interesado en la agilidad es el fútbol. Tal es el caso de Vanderford et al. (2004), quienes evidenciaron mejoría en la agilidad y las habilidades específicas, a través de la edad, lo cual, se podría atribuir, posiblemente, a la madurez física. La participación femenina en este deporte se ha incrementado en los últimos años y se evidencia en las investigaciones encontradas (Vescovi et al. 2006), cuyos hallazgos muestran pequeñas diferencias de posición y el desempeño físico (agilidad) entre jugadoras de fútbol, por lo tanto, los entrenadores deben considerar todos los componentes relacionados con el alto rendimiento en este deporte.

Farrow et al. (2005) presentaron una nueva metodología para la medición de la agilidad en jugadores de Netball, respondiendo a una presentación de video interactivo de un jugador, iniciando un pase, comparándolo con un movimiento de agilidad pre-planeado donde no hubo estímulo externo. El hallazgo clave en esta investigación es que las pruebas de agilidad planeada no suministran una indicación verdadera del desempeño del atleta en un deporte de conjunto, donde muchas de las respuestas de agilidad ejecutadas por un jugador son la reacción a un oponente.

En el campo de la educación física, los investigadores han querido diseñar y validar pruebas de agilidad en la primaria (Fernández et al. 2006); los resultados demostraron una alta fiabilidad, un grado de dificultad bajo y en la duración de la prueba resultó económica. En otras pruebas empleadas como la que valora la agilidad en la batería Eurofit del año 1992, prima la velocidad de desplazamiento sobre otros componentes. Además, los COD, a pesar de ser constantes, se realizan de la misma manera y a intervalos excesivos. También, Martínez et al. (2003) y Grosser \& Starischka (1988) incluyen en sus protocolos pasar por debajo de las vallas y saltar, situación que invalida la prueba para los niños de esta edad.

En el contexto nacional, aunque las investigaciones no se centran en la valoración de la agilidad, se destaca el estudio realizado en Medellín (Colombia), por Monroy
(1994), el cual, nos muestra la relación entre las capacidades de velocidad, de agilidad y de potencia de miembros inferiores, las cuales, se han venido trabajando con niños practicantes de tenis, encontrando una influencia significativa de la carga de entrenamiento sobre el desarrollo de la velocidad, pero no significativa sobre la agilidad ni la potencia de miembros inferiores.

En resumen, los problemas de la valoración de la agilidad que se han detectado, según el punto de vista de varios autores es que estos deberían ser independientes de la velocidad, ya que, inicialmente, fueron utilizados para valorar la capacidad de acelerar, desacelerar y re-acelerar (Buttifant et al. 1999). Existe pues, una carencia de investigaciones acerca de la agilidad y, concretamente, sobre su valoración que aclaren y analicen aspectos acerca de la validez, fiabilidad y especificidad de estas pruebas que encontramos en la literatura, en deportes que le atribuyen a esta capacidad gran importancia en su desarrollo y perfeccionamiento.

Teniendo como base estos antecedentes, el propósito principal de esta investigación fue analizar la validez, la fiabilidad y la especificidad de tres pruebas de agilidad (carrera de obstáculos, T-test y Buttifant), aplicados a tres grupos distintos. Adicionalmente, se analizó la influencia de factores, como iniciar una prueba por derecha o por izquierda, y la composición corporal de los sujetos en los resultados de las pruebas de agilidad.

\section{MATERIALES Y MÉTODOS}

Participaron 41 estudiantes de la Universidad de Ciencias Aplicadas y Ambientales U.D.C.A, de los cuales, 17 jugadores son de fútbol $(21,0 \pm 0,5$ años, $65,5 \pm 2,4 \mathrm{~kg}$, $1,75 \pm 0,02 \mathrm{~m})$, diez, jugadores de Voleibol $(21,0 \pm 0,4$ años, $72,5 \pm 3,5 \mathrm{~kg}, 1,81 \pm 0,03 \mathrm{~m})$ y 14 estudiantes de la facultad de Ciencias del Deporte $(24,1 \pm 0,7$ años, $63,9 \pm 2,1 \mathrm{~kg}, 1,71 \pm 0,01 \mathrm{~m}$ ), de Bogotá (Colombia) Todos fueron varones saludables, quienes estuvieron compitiendo en el torneo universitario seccional Bogotá (selecciones de fútbol y voleibol) y dieron por escrito su consentimiento, antes de iniciar el estudio.

De los 41 sujetos que participaron en el presente estudio, 37 eran diestros, fueron valorados antropométricamente y realizaron tres pruebas de agilidad y una prueba de velocidad. Todas las pruebas fueron cronometradas manualmente, mediante el cronómetro Casio Lap 
memory 10. Las tres pruebas de agilidad eran diferentes y una de ellas específica para cada grupo, se repitió por segunda vez, con una diferencia de dos días. Las pruebas, se desarrollaron en dos sesiones diferentes y, adicionalmente en una tercera sesión, cada grupo repitió su prueba específica. El orden fue el siguiente: primera sesión, la prueba general y una específica y segunda, la otra prueba específica y una de velocidad. Antes del desarrollo de las pruebas, se realizó un calentamiento que incluía dos ensayos de las pruebas, una por derecha y otra por izquierda. Todos los grupos repitieron su prueba así: el grupo de estudiantes de la facultad de ciencias del deporte repitió la prueba carrera de obstáculos (OBS), los practicantes de fútbol repitieron la prueba de Buttifant (BUT) y los practicantes de voleibol repitieron el T-test. Se seleccionaron las siguientes pruebas de agilidad y velocidad:

La prueba OBS: se desarrolló utilizando dos vallas a una altura de $90 \mathrm{~cm}$. enfrentadas y separadas $6 \mathrm{~m}$, con dos banderas ubicadas a $3 \mathrm{~m}$ y enfrentadas $4 \mathrm{~m}$. El sujeto salía de pie y describía una "X" pasando por debajo de la primera valla, posteriormente pasaba por encima de la segunda valla y, finalmente, por encima de la valla inicial. Se realizaron dos intentos por izquierda y por derecha, obteniendo el mejor tiempo como representativo de ambas mediciones, en tanto que se llevaron a cabo manualmente.

La prueba BUT (Buttifant et al. 2002): se realizó colocando seis picas de madera de $1,3 \mathrm{~m}$ de altura, separadas entre sí $4 \mathrm{~m}$ y determinando un recorrido en zigzag de $20 \mathrm{~m}$. La separación lateral de las picas fue de $6 \mathrm{~m}$. El futbolista a la voz de preparados, listos ya, debía recorrer en el menor tiempo posible los $20 \mathrm{~m}$, con cambios de dirección. Se realizaron dos intentos, variando la situación de la primera pica a la izquierda y derecha de los sujetos, obteniendo así el mejor tiempo, registrándolo como representativo de ambas mediciones.

El T-Test (Badenhorst, 1998): se ubicaron cuatro conos separados 5m entre sí, formando la letra "T". El sujeto a la voz de preparados, listos ya, partía en línea recta a tocar con la mano el primer cono, luego seguía a tocar el cono de la izquierda regresaba al cono central, seguía al cono de la derecha y, finalmente, terminaba en el cono del inicio. Se realizaron dos intentos variando la dirección de izquierda o derecha del primer cono, obteniendo el mejor tiempo y la media de los dos intentos. Este último valor fue seleccionado como representativo de ambas mediciones, en tanto que se llevaron a cabo manualmente.

La prueba de Velocidad de 30m: se realizó con salida alta, con un pie pisando la línea de salida (justo en la mitad del pie) y el otro y los brazos en posición libre. No se permitieron balanceos hacia atrás, ni despegar el pie del suelo. El sujeto corrió a la máxima intensidad los 30m. Se hicieron tres intentos, obteniendo el mejor tiempo y la media de ellos. Este último valor fue seleccionado como representativo de las tres mediciones, en tanto que se llevaron a cabo manualmente.

Siguiendo las consideraciones del GREC (Esparza, 1993), se tomó una medición relativa del peso, talla y ocho pliegues cutáneos (tricipital, bicipital, subescapular, pectoral, supra espinal, abdominal, muslo y pierna), de los que se obtuvo el sumatorio de ocho pliegues (todos ellos) y el sumatorio de seis pliegues (tricipital, subescapular, supra espinal, abdominal, muslo y pierna). A partir del sumatorio de seis pliegues, se obtuvo el porcentaje de masa grasa, atendiendo a la ecuación de Yushaz (Esparza, 1993): \% Grasa $=($ Sumatorio seis pliegues $\times 0,097)+3,64$. A partir del peso y la talla de los participantes, se obtuvo el índice de masa corporal o Body Mass Index (BMI): BMI $=$ Peso $(\mathrm{kg}) /$ Talla2 $(\mathrm{m})$. El material utilizado consistió en una báscula digital de precisión 0,005kg (Mobba ${ }^{\circledR}$ ); tallímetro (Holtain $\left.{ }^{\circledR}\right)$ y compás de pliegues cutáneos de 0,2mm (Harpenden $\left.{ }^{\circledR}\right)$.

El tratamiento gráfico, se registró en la hoja de cálculo Excel-v7.0 y el tratamiento estadístico en el paquete Statistica-v4.5 para Windows. Los resultados, se muestran como media y error estándar de la media (Media $\pm E E M)$. Para el estudio de las diferencias entre los tres grupos analizados, se hizo un análisis de la varianza (ANOVA), aplicando una prueba mediante la prueba de Newman-Keuls; en el análisis de las diferencias en el mismo grupo, al aplicar dos veces la misma prueba, se ha utilizado un análisis de la varianza (ANOVA), para medida repetidas. Para el cálculo de las correlaciones entre las variables, se empleó el coeficiente de correlación de Pearson. Los niveles de significación "p" son: n.s. = no significativa ó $\mathrm{p}<0,05 ; *=\mathrm{p}<0,05 ;{ }^{*}=\mathrm{p}<0,01$; $* * *=p<0,001$. 


\section{RESULTADOS Y DISCUSIÓN}

Según los resultados la prueba de Buttifant utilizado, tradicionalmente en el deporte del fútbol parece beneficiar a los practicantes de fútbol y el T-Test usado, preferencialmente, en voleibol, también parece favorecer a los practicantes de este deporte (Figura 1). Los trabajos seleccionan las pruebas de agilidad por su parecido a los desplazamientos propios de la actividad deportiva, pero ninguno ha comprobado que el rendimiento puede ser mayor en unos u otros.

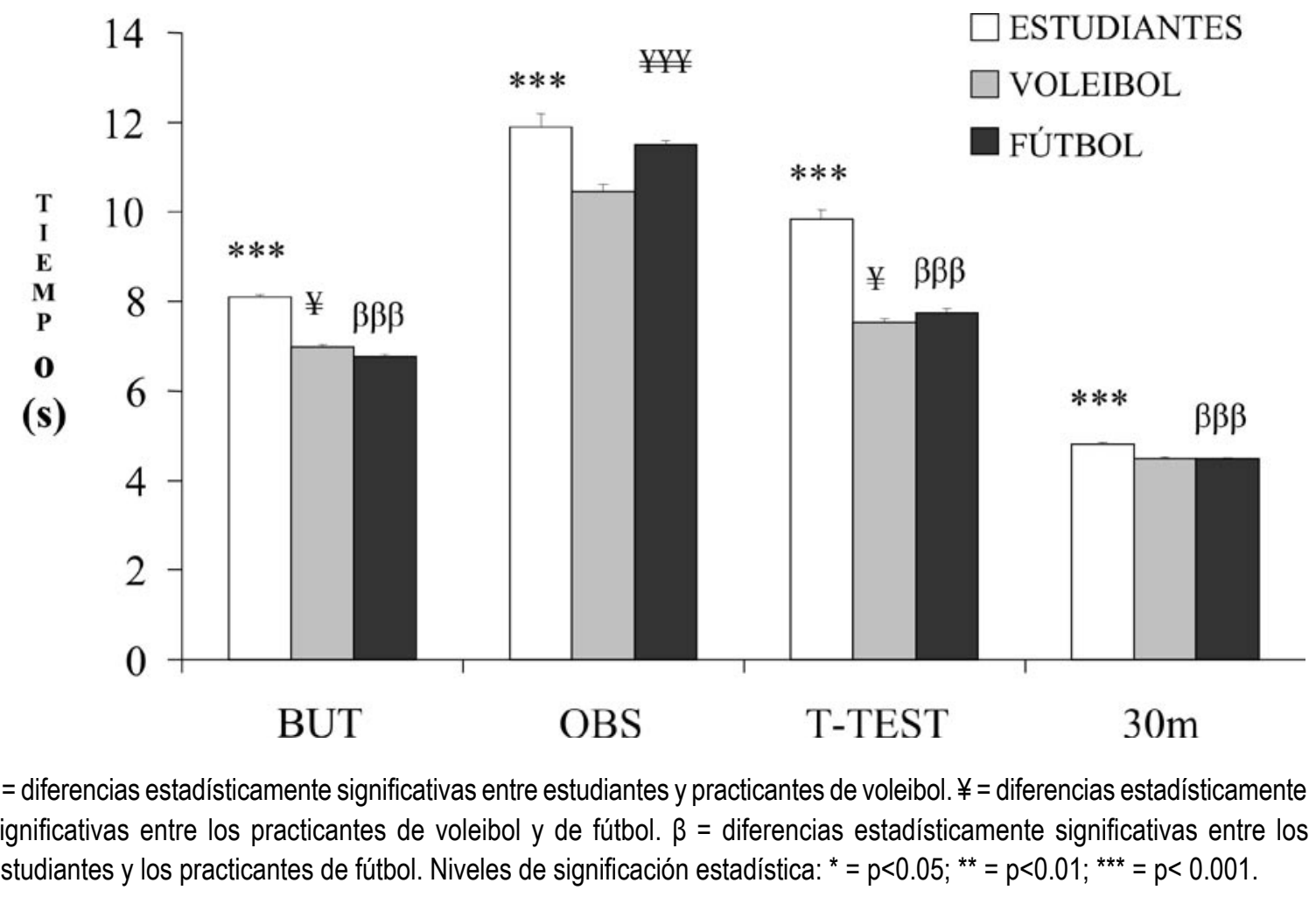

Figura 1. Diferencias encontradas entre los grupos de participantes en las pruebas de agilidad y la prueba de velocidad.

Con relación a la influencia de iniciar una prueba por derecha o por izquierda en la literatura se encontró que no hay claridad en este aspecto, ya que estudios realizados por Djevalikian (1993), citados por Sheppard \& Young (2006) quien examinó la relación entre los desequilibrios de fuerza en la potencia muscular concéntrica entre la pierna derecha e izquierda y los COD, mientras se aparta la pierna más débil, sus resultados sugirieron que no hubo ninguna relación significativa entre la potencia del músculo concéntrico y el COD. Sin embargo, Young et al. (2002) informaron que los desequilibrios musculares de las extremidades inferiores influyen en el COD. En el presente trabajo, no se obtienen diferencias significativas entre realizar las pruebas de agilidad por la derecha y por la izquierda, posiblemente, debido a que todas las pruebas siempre iniciaron por la derecha y siendo la mayoría de los ejecutantes diestros, podría haberles beneficiado en el aprendizaje de la prueba (Tabla 1). Por lo tanto, futuros trabajos deberían aleatorizar el inicio por la derecha e izquierda. Según el trabajo de Gabbett et al. (2006) es muy útil la aplicación del T-test para la selección de talentos en voleibol; sin embargo, según nuestros resultados, la fiabilidad del T-test en diferentes sesiones de valoración es la más baja $(r=0,79)$, a pesar de ser una prueba específica para los jugadores de este deporte (Figura 2B). Es necesario que futuros trabajos establezcan el número mínimo de sesiones de valoración con el T-test, para conseguir una fiabilidad mayor $(R>0,90)$. En este sentido, se debe tener en cuenta que los tiempos en las pruebas de agilidad 
se cronometraron manualmente y esto pudo haber afectado su fiabilidad. Sin embargo, se observa que la fiabilidad de las pruebas OBS y BUT fue muy alta en los practicantes de fútbol $(R>0,99)$, (Figura $2 A)$, lo que hace pensar que el cronometraje manual no fue determinante. No obstante, futuros trabajos deberían emplear sistemas de cronometraje más precisos (ejemplo células fotoeléctricas), para medir la agilidad. Teóricamente, factores como la grasa del cuerpo y las longitudes de segmentos corporales pueden contribuir al desempeño de la agilidad. Las opiniones han sido contradictorias y mientras unos autores han revelado que quienes rinden mejor en las pruebas de CODS de rugby y fútbol también tienden a tener menor grasa en el cuerpo (Gabbett, 2002; Meir et al. 2001; Reilly et al. 2000); otros autores han encontrado en jugadores de rugby una baja relación no significativa, entre ambas variables $(r=0,21)$ (Webb E Lander, 1983).

Tabla 1. Valores medios (Media $\pm E E M)$ de las pruebas de agilidad (BUT, OBS y T-TEST), realizados por el lado derecho e izquierdo.

\begin{tabular}{|c|c|c|}
\hline & Derecha & Izquierda \\
\hline BUT & $7,28 \pm 0,11$ & $7,28 \pm 0,11$ \\
\hline OBS & $11,26 \pm 0,15$ & $11,34 \pm 0,12$ \\
\hline T-TEST & $8,41 \pm 0,19$ & $8,40 \pm 0,19$ \\
\hline
\end{tabular}

A

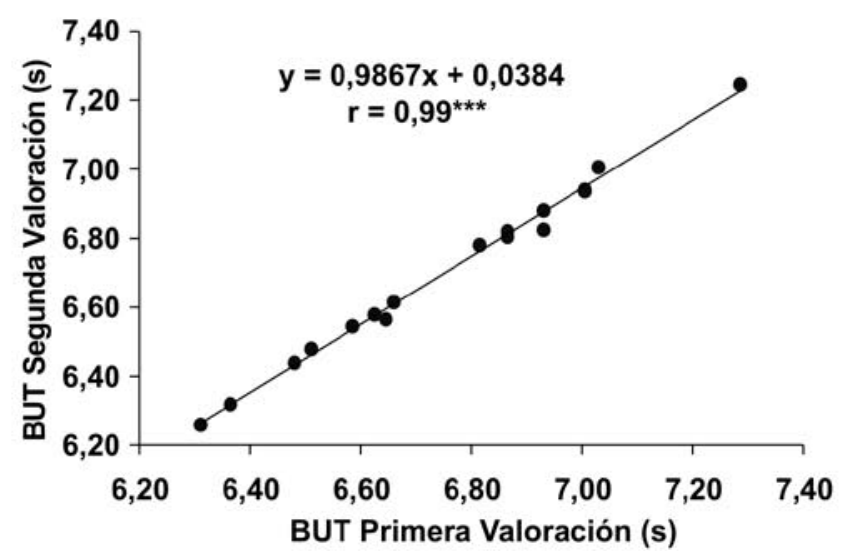

B

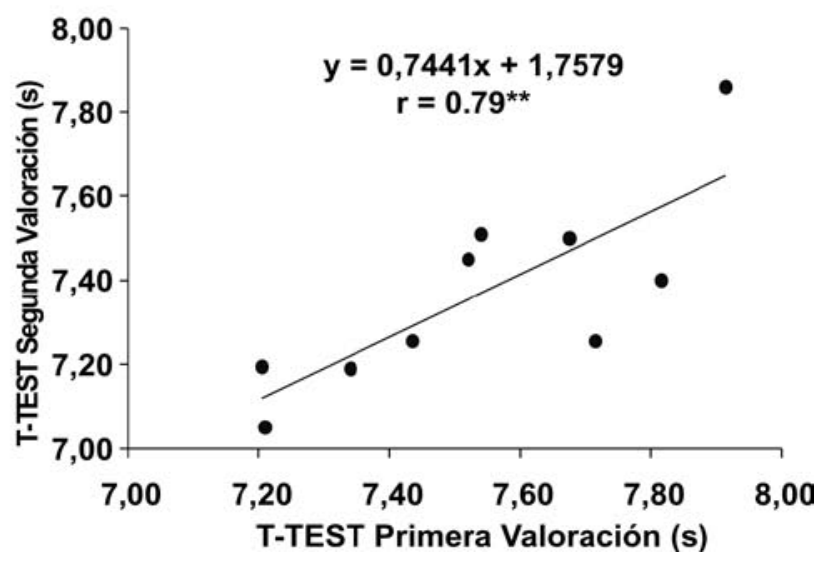

Figura 2. A. Correlación obtenida entre la primera y segunda valoración con el test BUT en practicantes de fútbol. Niveles de significación estadística: ${ }^{* *}=p<0,01 ;{ }^{* *}=p<0,001$. B. Diferencias entre la primera y segunda valoración con el T-TEST en practicantes de voleibol.

En el presente trabajo, no se encontraron relaciones entre las medidas de peso y talla y los resultados de las pruebas de agilidad; sin embargo, se hallaron relaciones entre el porcentaje graso y el rendimiento en dichas pruebas (Figura 3). Los sujetos con mayor grasa corporal tienden a rendir menos en las pruebas de agilidad. De hecho, incluso en un mismo equipo de élite (primera división de la liga española), estas capacidades se relacionaron negativamente con el porcentaje de grasa corporal (Villa et al. 2000), por lo tanto, es razonable afirmar que la composición corporal afecta a la agilidad y a las cualidades asociadas a la misma, como son la velocidad y/o resistencia.

Concretamente, el compartimiento de grasa corporal afecta negativamente al rendimiento en agilidad. Existen pocas investigaciones que han correlacionado estas variables. En general, en varios trabajos de investigación, el índice de masa corporal (IMC) no es un buen indicador de sobrepeso o adiposidad. Así se ha descrito en varios 

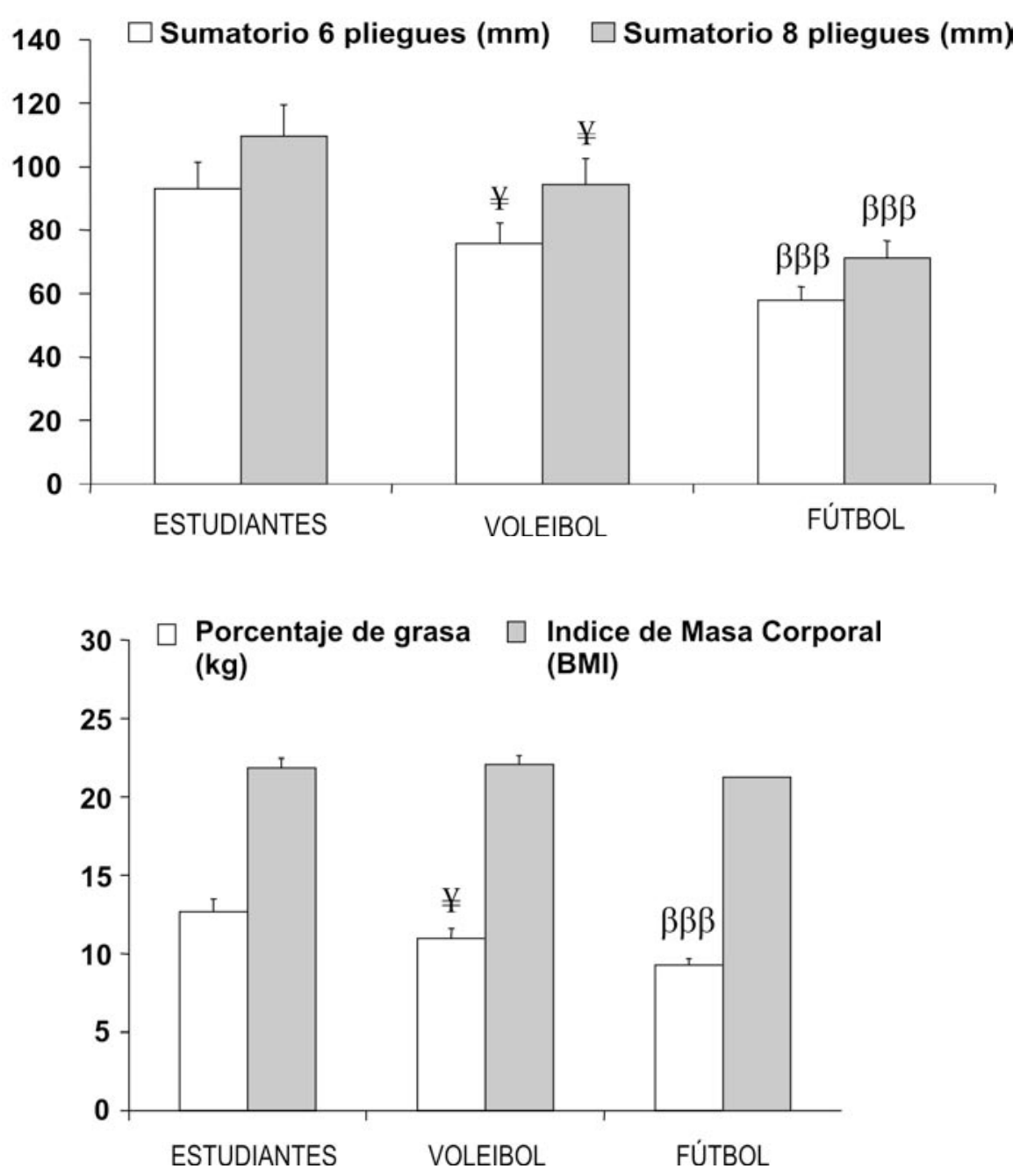

$¥=$ diferencias estadísticamente significativas entre los practicantes de voleibol y de fútbol. $\beta=$ diferencias estadísticamente significativas entre los estudiantes y los practicantes de fútbol. Niveles de significación estadística: $¥=p<0,05 ; \beta \beta \beta=p<0,001$.

Figura 3. Diferencias encontradas entre los grupos en las sumatorias de seis y ocho pliegues cutáneos, el porcentaje de grasa corporal y el índice de masa corporal.

estudios, como el de Bouchard (2000), en su revisión sobre composición corporal una de las limitaciones del IMC, como las tablas de talla y de peso, fallan al considerar la proporción de la composición corporal o como el más importante componente en la distribución de la grasa corporal. Finalmente, entre la relación de la agilidad y la velocidad, a pesar de que la prueba de OBS es considerado como una prueba específica para estudiantes, éste mostró una relación altísima con la prueba de $30 \mathrm{~m}$, tanto con los estudiantes como con los practicantes de voleibol, por lo que, posiblemente, no sea una prueba de agilidad y esté valorando también la velocidad. La prueba BUT no se relacionó con la velocidad, como indica Buttifant et al. (2002) (Tabla 2). Con relación al T-test ocurrió algún problema ligado al aprendizaje de los jugadores de voleibol (baja correlación entre valoraciones 1 y 2) (Figura 2B). Estos resultados, en general, confirman anteriores hallazgos, donde la aceleración, la velocidad máxima y la agilidad son cualidades distintas y, por lo tanto, los criterios de su valoración deberían serlo. 
Tabla 2. Correlaciones (Pearson) entre los tres tests de agilidad (BUT, OBS y T-TEST) y el test de velocidad de 30m. Niveles de significación estadística: ${ }^{* *}=p<0,01 ;{ }^{* * *}=p<0,001$.

\begin{tabular}{|c|c|c|c|}
\hline & OBS & T-TEST & $30 \mathrm{~m}$ \\
\hline BUT & $0,76^{* *}$ & $0,75^{* *}$ & $0,76^{* *}$ \\
\hline OBS & & $0,76^{* *}$ & $0,90^{* * *}$ \\
\hline T-TEST & & & $0,71^{* *}$ \\
\hline
\end{tabular}

\section{CONCLUSIONES}

La validez de las pruebas de agilidad depende, en gran manera, del concepto de agilidad que se maneje y también de las características particulares de cada deporte. Este es el primer trabajo que demuestra, experimentalmente, que el rendimiento en una prueba de agilidad depende de la modalidad deportiva. Siguiendo la opinión de Buttifant et al. (2002), la única prueba de agilidad que ha resultado válida en la presente investigación es la prueba que lleva su nombre.

La fiabilidad de las pruebas ha sido muy alta para OBS y BUT, pero no aceptable para T-test. Es posible que esta prueba sea demasiado inespecífica, incluso para personas que no practican ninguna actividad deportiva. No obstante, todas las pruebas mostraron una tendencia a la mejora en la segunda valoración, por lo que futuros trabajos deberían establecer el número de sesiones de evaluación necesarias para considerar que el rendimiento de la prueba se ha estabilizado. Normalmente, se diseñan las pruebas de agilidad intentando simular los desplazamientos específicos de un deporte; sin embargo, muy pocos estudios demuestran, experimentalmente, que esa prueba sea específica para un deporte. En el presente estudio, se demostró que la prueba de BUT es más específica para los practicantes de fútbol, el T-test para los de voleibol, y la prueba de OBS ha resultado igual de inespecífica para todos los grupos analizados.

No se ha podido establecer una influencia de la dominancia lateral en la realización de las pruebas de agilidad por la derecha o por la izquierda y se puede deber a que no se hizo aleatorio este orden a la hora de ejecutar la prueba, pudiendo haber influido el "factor aprendizaje" de la misma.

La agilidad es un compendio de cualidades coordinativas y otras capacidades físicas básicas. Por su relación con estas últimas, se ha mostrado sensible a la composición corporal de los sujetos evaluados, demostrándose en todos los grupos que un mayor componente graso deterioraron los resultados en las pruebas.

\section{BIBLIOGRAFÍA}

BADENHORST, E. 1998. 'n Keuringsmodel vir talentidentifisering by 16 -jarige sokkerspelers. [A selection model for talent identification for 16-year-old soccer players. South Africa.] Master's thesis, North West Univ. 52p.

BAKER, D. 1999. A comparison of running speed and quickness between elite professional and young rugby league players. J. Strength Cond. Coach. 7(3):3-7.

BARNES, J.; SCHILLING, B.; FALVO, M.; WEISS, L. 2007. Relationship of jumping and agility performance in female volleyball athletes. J. Strength Cond. Res. 21(4):1192-1196.

BARROW, H.; McGEE, R. 1971. A practical approach to measurement in physical education. Philadelphia, PA: Lea \& Febiger. p.175-178.

BLOOMFIELD, J.; ACKLAND, T.R.; ELLIOT, B.C. 1994. Applied anatomy and biomechanics in sport. Melbourne, VIC: Blackwell Scientific. p.307-310.

BOUCHARD, C. 2000. Interview composition, energy balance and weight control. Human Kinetics (Champaign, IL) Cap 28, p.752.

BUTTIFANT, D.; GRAHAM, K.; CROSS, K. 1999. Agility and speed in soccer players are two different performance parameters. Paper presented at the 
Science and Football IV Conference, Sydney, NSW. p.809-811.

BUTTIFANT, D.; GRAHAM, K.; CROSS, K. 2002. Agility and speed in soccer players are two different performance parameters. In: Science and Football IV. W.Spinks, ed. London: Routledge, p.329-332.

DRAPER, J.A.; LANCASTER, M.G. 1985. The 505 test: a test for agility in the horizontal plane. Austral. J. Science Medicine Sport. 17(1):15-18.

ESPARZA, F. 1993. Manual de cineantropometria. Madrid: FEMEDE. p.95-112.

FARROW, D.; ABERNETHY, B.; JACKSON, R.C. 2005. Probing expert anticipation with the temporal occlusion paradigm: Experimental investigations of some methodological issues. Motor Control. 9:332-351.

FERNÁNDEZ, I.; BAHAMON, J.; MÉNDEZ, D. 2006. Validación de un test de agilidad, adaptado a las características anatómico-fisiológicas y posibilidades motrices del niño en primaria apto para la valoración global de la capacidad motriz del alumno. Rev. Ciencias de la Actividad Física y del Deporte. 15:1-14.

GABBETT, T. 2002. Influence of physical characterstics on selection in a semi professional rugby league team: A case study. J. Sports Sci. 20:399-405.

GABBETT. T.; GEORGIEFF, B.; ANDERSON, S.; COTTON B. 2006. Changes in skill and physical fitness following training in talent- identified volleyball players. J. Strength Cond. Res. 20(1):29.

GROSSER, M.; STARISCHKA, S. 1988. Test de la condición física. Ed.Martínez Roca. Barcelona. p.242-243.

MARTÍNEZ, E.J.; ZAGALA, ML., LINARES, D. 2003. Las pruebas de aptitud física en la valoración de la condición física en la ESO. Apunts, 71:8-13.

MEIR, R.; NEWTON, R.; CURTIS, E.; FARDELL, M.; BUTLER, B. 2001. Physical fitness qualities of professional rugby league football players:Determination of positional differences. J. Strength Cond. Res. 15:450-458.
MONROY, H. 1994. Influencia de la carga de entrenamiento físico sobre el desarrollo de las capacidades velocidad, agilidad y potencia de miembros inferiores en niños tenistas infantiles. Universidad de Antioquia. ITF coaching. p.1-20.

REILLY, T., WILLIAMS, A. M., NEVILL, A., Y FRANKS, A. 2000. A multidisciplinary approach to talent identification in soccer. J. Sport Sci. 18:695-702.

SHEPPARD, J.; YOUNG, W. 2006. Agility literature review: Classifications, training and testing. (PrEview article). J. Sports Sci. 24(1):1-14.

TSITSKARSIS, G.; THEOHAROPOULUS, A.; GAREFIS, A. 2003. Speed,speed dribble and agility of male basketball players playing in different positions. J. Human Movement Studies. 45:21-30.

VANDERFORD. M.L.; MEYERS, M.C.; SKELLY, W.; STEWART HAMILTON, K. 2004. Physiological and sport- specific skill response of olympic youth soccer athletes. J. Strength Cond. Res. 18(2):334-342.

VESCOVI, J.D., BROWN, T.D., MURRAY, T.M. 2006. Positional characteristics of physical performance in division I college female soccer players. J. Sport Med. Phys . Fitness. 46(2):221-225.

VILLA, J.G., GARCÍA, J., MORENO, C. 2000. Influencia de una pretemporada en el perfil cineantropométrico de futbolistas. Arch. Medicina del Deporte. 17(75):9-20.

WEBB, P.; LANDER, J. 1983. An economical fitness testing battery for highschool and college rugby teams. Sports Coach. 7(3):44-46.

YOUNG, W.B.; HAWKEN, M.; McDONALD, L. 1996. Relationship between speed, agility, and strength qualities in Australian rules football. J. Strength Cond. Coach. 4(4):3-6.

YOUNG, W.B.; JAMES, R.; MONTGOMERY, I. 2002. Is muscle power related to running speed with changes of direction? J. Sports Medicine and Phys. Fitness. 43:282-288.

Recibido: Febrero 21 de 2008

Aceptado: Octubre 18 de 2008 